\title{
Prescription Pattern in the Management of Secondary Prevention of Stroke in Post Stroke Patients
}

\author{
Jisha Annie ${ }^{1,{ }^{*}}$, RC Doijad ${ }^{1}$, Emmanuel James ${ }^{2}$ \\ 'Department of Pharmacy Practice, Krishna Institute of Pharmacy, Krishna (Deemed to be University), Karad, Maharashtra, INDIA. \\ 2Department of Pharmacy Practice, Amrita School of Pharmacy, Amrita Vishwa Vidyapeetham University, AIMS Health Sciences Campus, Kochi, Kerala, INDIA.
}

\begin{abstract}
Background: To analyze the drug prescription pattern for secondary prevention of stroke, medication adherence, knowledge and awareness about the disease, direct medical cost involved in the treatment of stroke. Methods: A prospective interventional study was done on ischemic stroke patients for a period of 10 months in the outpatient department of stroke after obtaining ethical clearance. Medical prescriptions of all patients were checked as per the ASA guidelines. Educational interventions were given on lifestyle modifiable risk factors with the aid of booklets on stroke. All the patients were assessed for knowledge on stroke, medication adherence at baseline and post-intervention using validated questionnaires. Direct medical cost for treatment during the study was estimated. Results: The mean age of study patients was $59.69 \pm 9.56$ years and $67 \%$ were males. Disease knowledge, medication adherence improved significantly postintervention $(P<0.001)$. Among the various classes of drugs prescribed for secondary stroke prevention, antihypertensive medications constituted the largest class $(81 \%)$ followed by antiplatelets and statins $(68 \%)$. Out of 100 patients 99 were having $\mathrm{CHADS}_{2}$ score $\geq 2,67 \%$ were prescribed
\end{abstract}

with antithrombotic medications who were having medium to high risk for secondary stroke. The mean medication cost for the treatment of stroke during the study period was $33,077.35 \pm 10241.32$ Rs. Conclusion: The data procured from this study highlights the importance of prescribing patients with anticoagulants rather than antithrombotic or combination of antithrombotic in those who are at medium to high risk for future strokes. Clinical pharmacist's contributions can provide better management in secondary prevention of stroke.

Key words: Anticoagulants, Clinical pharmacist, Medication adherence, Secondary prevention, Stroke.

\section{Correspondence}

Mrs. Jisha Annie

Department of Pharmacy Practice, Krishna Institute of Pharmacy, Krishna (Deemed to be University), Karad, Maharashtra, INDIA.

Email id: jishaannie22@gmail.com

DOI: 10.5530/ijpi.2021.4.77

\section{INTRODUCTION}

Stroke the second leading cause of death and third leading contributor to disability-adjusted life-years in the world. ${ }^{1,2}$ Among patients who survive an acute stroke, the risk of stroke recurrence is highest within the first few weeks, approximately 10\% in the first year and 5\% per year subsequently. ${ }^{3}$ Even though advanced treatment for acute ischemic stroke is available nowadays, the number of effective and feasible treatments remains limited. ${ }^{4,5}$ The economic impact of stroke will vary from different countries. For this reason, secondary prevention of ischemic stroke remains a major clinical and public health issue. ${ }^{6}$ Recurrent stroke can be prevented to a great extent by adopting evident based treatment strategies, improving medication adherence and by management of modifiable risk factors. Various studies have proved the benefits of including a clinical pharmacist in the patient care team in improving medication adherence, management of hypertension and dyslipidemia which are the major modifiable risk factors targeted in secondary prevention of stroke. ${ }^{7-9}$ Hence this study aimed to explore the impact of clinical pharmacist interventions in Indian population in secondary stroke prevention management and to assess the prescription pattern of medicines used for secondary stroke prevention.

\section{MATERIALS AND METHODS}

\section{Participant selection}

A Prospective interventional study was done for a period of 10 months in the out-patient (OP) department of stroke division, Amrita Institute of Medical Sciences and Research Centre, Kochi, a 1250 bedded tertiary care teaching hospital after approval of the institutional review board.

\section{Inclusion Criteria}

All stroke OP patients who satisfied the inclusion and exclusion criteria were selected for the study process. Stroke patients aged $\geq 18$ years admitted under stroke department and discharged from stroke unit, including those who were started on oral anticoagulants were included.

\section{Exclusion Criteria}

Pregnant and breast-feeding patients, those with severe liver/renal insufficiency, those on long term follow up prior to the commencement of the study or patients and /or their caregivers unwilling to provide written informed consent were excluded.

Relevant medical data were obtained by personal interview of communication with patients and/or their caregivers, examination of electronic medical records and assessment using validated questionnaires.

\section{Secondary stroke prevention}

Patients were evaluated on their first visit after discharge ( $7 \pm 3$ days) for baseline knowledge and awareness regarding the disease using locally prepared validated questionnaire and medication adherence using Morisky Medication Adherence 8-item scale. ${ }^{10-12}$ and reassessed during follow up visit. The patients were provided with stroke education 
booklets at their baseline visit and made them aware regarding the use of 'pill box'. From discharge onwards proper medication counseling was given and all the medications prescribed were checked at each visit. The direct medical cost was tabulated which includes consultation cost, medication cost, lab investigation cost at each visit.

One hundred prescriptions of all the patients were assessed and their prescription pattern was compared with standard guidelines of American Stroke Association. The risk of the patients for future stroke was assessed by $\mathrm{CHADS}_{2}$ Score and CHADVASc score.

Patients on oral anticoagulants were given additional educational interventions and booklets on oral anticoagulation. Ischemic stroke in patients with atrial fibrillation were assessed using $\mathrm{CHADS}_{2}$ as well as $\mathrm{CHA}_{2} \mathrm{DS}_{2}$-VASc score. Bleeding risk in patients on oral anticoagulants were assessed using HAS-BLED score. The safety and effectiveness of oral anticoagulants including newer oral anticoagulant dabigatran prescribed to the patients were evaluated by monitoring the ADRs and by the occurrence of secondary stroke respectively.

\section{Determinant measures}

Various patient level factors were studied viz.

i) Demographic characteristics - Age, sex, ethnicity, and education level. ii) Co-morbidities and prescriptions recorded prior to the stroke event: previous hypertension, diabetes, lipidemia, heart related problems, previous stroke, or transient ischemic attack (TIA) events and prior prescriptions of antiplatelet drugs, anticoagulant drugs, antihypertensive drugs, and lipid lowering drugs.

iii) Lifestyle - Obesity, smoking, alcohol addiction etc.

iv) Patients disability status upon discharge was assessed by using specific scale.

\section{Statistical Analysis}

The medication cost for both patients taking warfarin and dabigatran was calculated each time. For patients on warfarin, the cost for doing INR test was also included. The data were tabulated using Microsoft excel and statistical analysis was done using Statistical package for the Social Sciences (SPSS) software version 21. The significance was compared using $\mathrm{Z}$ test and Wilcoxon signed rank test.

\section{RESULTS}

Out of 100 ischemic stroke patients selected, $67 \%$ were males and the mean age of study patients was $59.69 \pm 9.56$ years.

Table 1 and 2 shows the $\mathrm{CHADS}_{2}$ and $\mathrm{CHA}_{2} \mathrm{DS}_{2-}$ VAScscore of patients in the study sample respectively. $99 \%$ patients were found to have $\mathrm{CHADS}_{2}$ score $\geq 2$ points and $99 \%$ had $\mathrm{CHA}_{2} \mathrm{DS}_{2}$ VAScscore $\geq 2$ points. The general pattern of prescription of drugs for the secondary prevention of stroke is shown in Table 3. 68\% of study population was prescribed with antiplatelet drugs, $47 \%$ with anticoagulants, $81 \%$ with antihypertensives, $68 \%$ with stains for secondary stroke prevention. The knowledge and awareness of patients regarding stroke improved significantly postintervention. Only $2 \%$ of the patients were aware of the sudden warning signs of stroke at baseline and it improved to $98 \%$ post-intervention. The responses of our study patients to various questions on knowledge and awareness regarding stroke is shown in Table 4.

The medication adherence of patients improved significantly postintervention $(p<0.001)$ (Figure 1$)$ and the direct medical cost for the treatment of study patients during the study period was estimated and is shown in Table 5.

Bleeding risk in patients on oral anticoagulants was assessed using HASBLED scoring. HAS-BLED scoring of patients on oral anticoagulants
Table 1: CHADS $_{2}$ score of patients in the study sample $(n=100)$.

\begin{tabular}{ccc}
\hline CHADS $_{2}$ Score & \% of annual stroke risk* & No. (\%) of patients \\
\hline 0 & 1.9 & $1(1)$ \\
1 & 2.8 & $0(0)$ \\
2 & 4.0 & $3(3)$ \\
3 & 5.9 & $32(32)$ \\
4 & 8.5 & $56(56)$ \\
5 & 12.5 & $8(8)$ \\
Mean score & & 3.66 \\
SD & & 0.755 \\
\hline
\end{tabular}

* The chances for future stroke can be predicted by calculating $\mathrm{CHADS}_{2}$ score. Standard values representing $\%$ of annual stroke risk in the study patients.

Table 2: $\mathrm{CHA}_{2} \mathrm{DS}_{2}$ VASc score of patients in the study sample ( $\left.n=100\right)$.

\begin{tabular}{cc}
\hline $\mathrm{CHA}_{2} \mathrm{DS}_{2}$ VASc score & No. (\%) of patients \\
0 & 0 \\
1 & 1 \\
2 & 35 \\
3 & 12 \\
4 & 23 \\
5 & 21 \\
6 & 3 \\
7 & 4 \\
8 & 1 \\
9 & 0 \\
Mean & 3.58 \\
SD & 1.519 \\
\hline
\end{tabular}

Additional risk factors like vascular disease, female sex, age 64-75 are considered in $\mathrm{CHA}_{2} \mathrm{DS}_{2}$-VASc score

Table 3: General pattern of prescription of drugs for the secondary prevention of stroke.

\begin{tabular}{|c|c|c|}
\hline Drug categories & $\begin{array}{c}\text { No. }(\%) \text { of } \\
\text { patients }\end{array}$ & $\begin{array}{c}\text { Percentage of } \\
\text { medications }(n=504)\end{array}$ \\
\hline - $\quad$ Antiplatelets* & $68(68 \%)$ & 13.42 \\
\hline - $\quad$ Aspirin & $34(34 \%)$ & 6.746 \\
\hline - $\quad$ Clopidogrel & $9(9 \%)$ & 1.78 \\
\hline - $\quad$ Aspirin and Clopidogrel & $25(25 \%)$ & 4.96 \\
\hline - $\quad$ Anticoagulants ${ }^{*}$ & $47(47 \%)$ & 9.32 \\
\hline - Warfarin & $37(37 \%)$ & 7.34 \\
\hline - Dabigatran & $10(10 \%)$ & 1.98 \\
\hline - Antihypertensives & $81(81 \%)$ & 20.23 \\
\hline - Calcium channel blockers & $49(49 \%)$ & 9.72 \\
\hline - $\quad$ Angiotensin receptor blockers & $25(25 \%)$ & 4.96 \\
\hline - $\quad$ Beta blockers & $12(12 \%)$ & 2.38 \\
\hline - $\quad$ Alpha blockers & $11(11 \%)$ & 2.18 \\
\hline - Diuretics & $5(5 \%)$ & 0.99 \\
\hline - Dyslipidemics & & \\
\hline Statins & $68(68 \%)$ & 13.49 \\
\hline
\end{tabular}

The general prescription pattern of drugs for secondary stroke prevention in the study group is described and the highly prescribed class of drug is Antihypertensive. This prescription pattern was compared with the standard guidelines. 


\section{Table 4: Response of study patients to Knowledge and awareness questionnaire.}

\begin{tabular}{|c|c|c|c|c|}
\hline \multirow[t]{2}{*}{ SL. No. } & \multirow[b]{2}{*}{ Questions } & \multicolumn{2}{|c|}{$\%$ of patients giving correct responses } & \multirow[t]{2}{*}{ Z-value } \\
\hline & & Pre-intervention & Post-intervention & \\
\hline 1. & What do you understand by the term 'stroke'? & $3 \%$ & $94 \%$ & $48.49^{* *}$ \\
\hline 2. & What are the warning signs of stroke? & $2 \%$ & $98 \%$ & $33.20^{* *}$ \\
\hline 3. & Which hemisphere of the brain is affected by stroke? & $4 \%$ & $96 \%$ & $70.00^{* *}$ \\
\hline 4. & Which is the most common form of stroke? & $0 \%$ & $98 \%$ & $43.05^{* *}$ \\
\hline 5. & What is a hemorrhagic stroke? & $2 \%$ & $97 \%$ & $16.54^{* *}$ \\
\hline 6. & Name a risk factor for hemorrhagic stroke? & $12 \%$ & $88 \%$ & $21.34^{* *}$ \\
\hline 7. & What should you do if you find a person having symptoms of stroke? & $18 \%$ & $100 \%$ & $15.65^{* *}$ \\
\hline 8. & Is there a chance for second stroke after the first one? & $29 \%$ & $100 \%$ & $14.81^{* *}$ \\
\hline 9. & How can one prevent the recurrence of stroke? & $24 \%$ & $95 \%$ & $31.27^{* *}$ \\
\hline 10. & Man or woman who is at high risk for stroke? & $7 \%$ & $98 \%$ & $25.87^{* *}$ \\
\hline 11. & Smoker or ex-smoker who is at high risk for stroke? & $13 \%$ & $100 \%$ & $7.33^{* *}$ \\
\hline 12. & Name a modifiable risk factor of stroke? & $26 \%$ & $72 \%$ & $17.64^{* *}$ \\
\hline 13. & How can one prevent the extent of brain damage due to stroke? & $12 \%$ & $90 \%$ & $48.99^{* *}$ \\
\hline 14. & Which is the most powerful blood thinning drug used in prevention of stroke? & $0 \%$ & $96 \%$ & $48.49^{* *}$ \\
\hline
\end{tabular}

** Significant at 0.01 level, $\mathrm{P}<0.001$ indicates a significant difference in the knowledge score of study patient group before and after intervention.

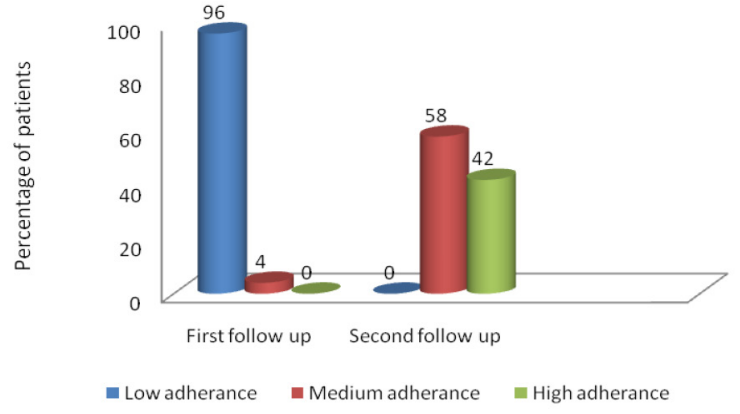

Figure 1: Medication adherence of study patients as per MMAS-8 Scale $(n=100)$.

both Warfarin and Dabigatran was assessed and 100\% of the study patients taking either warfarin or dabigatran had a HAS-BLED score $\leq 2$.

\section{DISCUSSION}

In our study we found a significant improvement in the stroke risk by the control of modifiable risk factors. This was achieved by the regular medical follow up along with clinical pharmacist's interventions in making the patients aware regarding the disease signs and symptoms, management of modifiable risk factors, improving medication adherence. About $8 \%$ of the people who had a primary stroke are likely to have stroke recurrence soon. ${ }^{13}$ So, prevention of stroke recurrence will play a key role in improving the overall health as well as economic burden of the patients.

Out of 100 patients selected for the study $67 \%$ were males and the remaining females which was like the group of patients in the study conducted by Pandian et al. ${ }^{14}$ The mean age group of our patients (59.69 \pm 10.15 years) compares well with the above-mentioned study group ( $59.7 \pm 14.1$ years).

The chances for future stroke can be predicted by calculating $\mathrm{CHADS}_{2}$ score. Patients having $\mathrm{CHADS}_{2}$ score 2 or more will have high risk for
Table 5: Direct medical cost estimation for stroke treatment in study patients $(n=100)$.

\begin{tabular}{|c|c|c|}
\hline Parameters & Total cost (Rs) & Mean cost (Rs) \\
\hline Consultation cost for first visit & 20,000 & $200 \pm 0.00$ \\
\hline $\begin{array}{l}\text { Cost of medications during hospital } \\
\text { stay }\end{array}$ & $3,74,275.76$ & $3,742.76 \pm 934.71$ \\
\hline Lab cost during hospital stay & $13,57,920$ & $13,579.2 \pm 3465.86$ \\
\hline $\begin{array}{l}\text { Consultation cost for follow up after } \\
\text { discharge }\end{array}$ & 40,000 & $400 \pm 0.00$ \\
\hline $\begin{array}{l}\text { Cost of medications for } 10 \text { months } \\
\text { after discharge }\end{array}$ & $11,79,179$ & $11,791.79 \pm 358.67$ \\
\hline $\begin{array}{l}\text { Lab cost for } 10 \text { months after } \\
\text { discharge }\end{array}$ & $3,36,360$ & $3,363.60 \pm 758.95$ \\
\hline Grand total & $33,07,734.76$ & $33,077.35 \pm 10241.32$ \\
\hline
\end{tabular}

The mean medical cost calculated by taking in to account of consultation cost, medical cost and lab costs during the study period are listed.

future stroke. ${ }^{15}$ Ninety nine percent of our study patients had CHADS score $\geq 2$ (Table 2). The additional risk factors that are expressed in the $\mathrm{CHA}_{2} \mathrm{DS}_{2}$-VASc have been proposed to complement the CHADS score. ${ }^{16}$ Additional risk factors like vascular disease, female sex, age 64-75 are considered in $\mathrm{CHA}_{2} \mathrm{DS}_{2}$-VASc score. So, this scoring truly identifies patients at higher risk for future stroke when compared to CHADS score. $\mathrm{CHADS}_{2}$ score ranges from 0-6 and $\mathrm{CHA}_{2} \mathrm{DS}_{2}$-VASc score ranges from 0 -9. In both scoring, patients having a score $\geq 2$ are grouped in high risk category.

According to American stroke association (ASA) guidelines for long term secondary prevention of stroke antiplatelets, anticoagulants, blood pressure lowering medications and lipid lowering drugs are given to patients. ${ }^{17}$ All our study patients received medications for secondary prevention of stroke. This was contradictory to the study done by Volpato ${ }^{18}$ where majority of patients were discharged without antithrombotic medications. In that study about $40 \%$ of the patients who had a previous stroke were neither receiving antiplatelets nor 
anticoagulants, but in our patients $68 \%$ were prescribed with antiplatelets who had an ischemic stroke, while the remaining 47 patients were given oral anticoagulants, 34 of these patients were given oral anticoagulants for stroke prevention in atrial fibrillation. More study patients could have been subjected to anticoagulation, but the confounding factors may be bleeding risk and old age, also the decision to start anticoagulants is solely doctor's decision in our health system. 15 patients were taking both anticoagulants and antiplatelets. Four among them were taking clopidogrel along with warfarin as they had undergone valve replacement surgery and the remaining were taking aspirin along with warfarin as they had coronary artery disease. All patients who had a previously treated hypertension as well as newly diagnosed one were taking appropriate class of antihypertensive medication as prescribed. Patients with dyslipidemia (total cholesterol levels are high) were prescribed with appropriate dose of statins to reduce the risk of stroke and cardiac complications. All patients who had uncontrolled diabetes were treated with oral hypoglycemic drugs and with insulin injections. All were educated regarding the lifestyle, dietary modification that can be followed to reduce the modifiable risk factors.

The knowledge of our study patients regarding the disease improved significantly $(P<0.001)$ post-intervention as compared to the baseline. This might be due to education given to the patients regarding the warning signs, risk factors of stroke, importance of early hospitalization and stroke rehabilitation measures etc. by clinical pharmacists. Our study had positive correlation with other studies conducted by Kothari et al. ${ }^{19}$ and Croquelois et al..$^{20}$ where the patients/caregivers had low knowledge regarding the warning signs and risk factors of stroke. Proper adherence to medications is very vital for secondary prophylaxis of stroke. Most patients had low medication adherence at baseline. Forgetfulness, fear about adverse drug effects, ignorance about the consequences of non-adherence may be the reasons for low adherence. Pharmacist led educational interventions can improve medication adherence. ${ }^{21}$ The mean adherence scores of patients improved significantly in the second follow up visits $(P<0.001)$. This might be due to patient counseling and patient education by clinical pharmacists.

HAS-BLED scoring was done to identify the bleeding risk of patients on oral anticoagulants. Patients having HAS-BLED score $\leq 2$ have low bleeding risk and they can be prescribed antithrombotic medications safely. A score $\geq 3$ indicates high risk for bleeding and these patients require review and monitoring of therapy. ${ }^{22}$ Traditional anticoagulant warfarin and newer anticoagulant dabigatran were used in our patients. As per our study results none of the patients on warfarin and dabigatran were having a high risk for bleeding at baseline.

On comparing newer anticoagulant Dabigatran with warfarin, number of patients using dabigatran (10) was less as compared to patients using warfarin (37) in our study where as in the non-inferiority trial conducted by Conolly ${ }^{23}$ comparing dabigatran and warfarin the total number of patients included was 18,113 and the follow up period was 2 years. Nevertheless, we compared the bleeding episodes in the two groups, and it was found to be non-significant $(P=0.284)$. As per our study dabigatran was not superior $(P=0.161)$ to warfarin $(13.51 \%)$ in preventing stroke recurrence which was in contradiction to the study obtained by Miller ${ }^{24}$ where it was found that newer anticoagulants were effective in reducing the risk for stroke and systemic embolism than warfarin. Another cohort study by Banerjee ${ }^{25}$ shows that when the risk for stroke and bleeding are high dabigatran, rivaroxaban and apixaban had a better clinical benefit than warfarin.

\section{CONCLUSION}

Educational interventions by clinical pharmacists can contribute to better disease knowledge and improved medication adherence. As per the
American Stroke Association guidelines the prescription pattern should be made rational according to the patient characteristics and $\mathrm{CHADS}_{2}$ score. The data procured from this study highlights the importance of prescribing patients with anticoagulants rather than antithrombotic or combination of antithrombotics in those who are at medium to high risk for future strokes. Antithrombotic or oral anticoagulants play an important role in preventing recurrence of cardio-embolic stroke. Clinical pharmacist's contributions can provide better management in secondary prevention of stroke.

\section{CONFLICT OF INTEREST}

The authors declare that there is no conflict of interest.

\section{REFERENCES}

1. Lozano R, Naghavi M, Foreman K, Lim S, Shibuya K, Aboyans V, et al. Global and regional mortality from 235 causes of death for 20 age groups in 1990 and 2010: A systematic analysis for the Global Burden of Disease Study 2010 Lancet. 2012;380(9859):2095-128. doi: 10.1016/S0140-6736(12)61728-0, PMID 23245604.

2. Murray CJ, Vos T, Lozano R, Naghavi M, Flaxman AD, Michaud $C$, et al. Disability-adjusted life years (DALYs) for 291 diseases and injuries in 21 regions, 1990-2010: A systematic analysis for the Global Burden of Disease Study 2010. Lancet. 2012;380(9859):2197-223. doi: 10.1016/S0140-6736(12)61689-4, PMID 23245608.

3. Gubitz G, Sandercock P. Prevention of ischaemic stroke. BMJ 2000;321(7274):1455-9. doi: 10.1136/bmj.321.7274.1455, PMID 11110741.

4. Broderick JP. Logistics in acute stroke management. Drugs. 1997;54;Suppl 3:109-16; discussion 116. doi: 10.2165/00003495-199700543-00015, PMID 9360858 .

5. Wardlaw JM, Yamaguchi T, Del Zoppo G. Thrombolytic therapy versus control in acute ischaemic stroke. In: Cochrane Collaboration. Cochrane Library. Issue 4. Oxford: Update [software]; 1999.

6. Mancia G, Grassi G. Secondary prevention of stroke: Old and new evidence. Aging Clin Exp Res. 2002;14(3):216-20. doi: 10.1007/BF03324440, PMID 12387531.

7. Sacco RL, Adams R, Albers G, Alberts MJ, Benavente O, Furie $K$, et al. American Heart Association, American Stroke Association Council on Stroke, Council on Cardiovascular Radiology and Intervention, American Academy of Neurology. Guidelines for prevention of stroke in patients with ischemic stroke or transient ischemic attack: a statement for healthcare professionals from the American Heart Association/American Stroke Association Council on Stroke: co-sponsored by the Council on Cardiovascular Radiology and Intervention: the American Academy of Neurology affirms the value of this guideline. Stroke. 2006;37(2):577-617. doi: 10.1161/01.STR.0000199147.30016.74, PMID 16432246.

8. Calis KA, Hutchison LC, Elliott ME, Ives TJ, Zillich AJ, Poirier T, et al. American College of Clinical Pharmacy. Healthy People 2010: Challenges, opportunities, and a call to action for America's pharmacists. Pharmacotherapy. 2004;24(9):1241-94. doi: 10.1592/phco.24.13.1241.38082. PMID 15460187.

9. Bond CA, Raehl CL, Franke T. Clinical pharmacy services and hospital mortality rates. Pharmacotherapy. 1999;19(5):556-64. doi: 10.1592/phco.19.8.556.31531 PMID 10331818.

10. Morisky DE, Ang A, Krousel-Wood M, Ward HJ. Predictive validity of a medication adherence measure in an outpatient setting. J Clin Hypertens (Greenwich). 2008;10(5):348-54. doi: 10.1111/j.1751-7176.2008.07572.x, PMID 18453793.

11. Krousel-Wood MA, Islam T, Webber LS, Re RN, Morisky DE, Muntner P. New medication adherence scale versus pharmacy fill rates in seniors with hypertension. Am J Manag Care. 2009;15(1):59-66. PMID 19146365.

12. Voils $\mathrm{Cl}$, Hoyle $\mathrm{RH}$, Thorpe $\mathrm{CT}$, Maciejewski ML, Yancy WS. Improving the measurement of self-reported medication nonadherence: Response to Authors. J Clin Epidemiol. 2011;64(3):258-61. doi: 10.1016/j.jclinepi.2010.02.023.

13. Dhamoon MS, Sciacca RR, Rundek T, Sacco RL, Elkind MS: Recurrent stroke and cardiac risks after first ischemic stroke: the Northern Manhattan Study. Neurology 2006; 66: 641-6.

14. Pandian JD, Kalra G, Jaison A, Deepak SS, Shamsher S, SinghY, et al. Knowledge of stroke among stroke patients and their relatives in Northwest India. Neurol India. 2006;54(2):152-6; discussion 156. PMID 16804258.

15. Gage BF, Waterman AD, Shannon W, Boechler M, Rich MW, Radford MJ Validation of clinical classification schemes for predicting stroke: Results from the National Registry of atrial fibrillation. JAMA. 2001;285(22):2864-70. doi: 10.1001/jama.285.22.2864, PMID 11401607.

16. Ellis C, Egede LE. Ethnic disparities in stroke recognition in individuals with prior stroke. Public Health Rep. 2008;123(4):514-22. doi: 10.1177/003335490812300413, PMID 18763414. 
17. Diener H, Albers G. Secondary stroke prevention Brainin M, Heiss WD (Eds.), Textbook of Stroke Medicine (3rd ed.), Cambridge University Press, Cambridge, United Kingdom (2019), pp. 410-42

18. Volpato S, Maraldi C, Blè A, Ranzini M, Rita Atti AR, Dominguez LJ, et al. Gruppo Italiano di Farmacoepidemiologia nell'Anziano (GIFA). Prescription of antithrombotic therapy in older patients hospitalized for transient ischemic attack and ischemic stroke: the GIFA Study. Stroke. 2004;35(4):913-7. doi: 10.1161/01.STR.0000121648.74433.b5, PMID 15001787.

19. Kothari R, Sauerbeck L, Jauch E, Broderick J, Brott T, Khoury J, et al. Patients awareness on stroke signs, symptoms, and risk factors. Stroke. 1997;28(10):1871-5. doi: 10.1161/01.str.28.10.1871, PMID 9341687.

20. Croquelois A, Bogousslavsky J. Risk awareness and knowledge of patients with stroke: Results of a questionnaire survey 3 months after stroke. J Neurol Neurosurg Psychiatry. 2006;77(6):726-8. doi: 10.1136/jnnp.2005.078618, PMID 16549417.

21. Bajorek BV, Krass I, Ogle SJ, Duguid MJ, Shenfield GM. Optimizing the use of antithrombotic therapy for atrial fibrillation in older people: A pharmacistled multidisciplinary intervention. J Am Geriatr Soc. 2005;53(11):1912-20. doi: 10.1111/j.1532-5415.2005.53564.x, PMID 16274372.

22. Pisters $R$, Lane DA, Nieuwlaat $R$, de Vos CB, Crijns HJ, Lip GY. A novel userfriendly score (HAS-BLED) to assess 1-year risk of major bleeding in patients with atrial fibrillation: The euro Heart Survey. Chest. 2010;138(5):1093-100. doi: 10.1378/chest.10-0134, PMID 20299623.

23. Connolly SJ, Ezekowitz MD, Yusuf S, Eikelboom J, Oldgren J, Parekh A, et al. RE-LY Steering Committee and Investigators. Dabigatran versus warfarin in patients with atrial fibrillation. N Engl J Med. 2009;361(12):1139-51. doi: 10.1056/NEJMoa0905561, PMID 19717844

24. Miller CS, Grandi SM, Shimony A, Filion KB, Eisenberg MJ. Meta-analysis of efficacy and safety of new oral anticoagulants (dabigatran, rivaroxaban, apixaban) versus warfarin in patients with atrial fibrillation. Am J Cardiol. 2012;110(3):453-60. doi: 10.1016/j.amjcard.2012.03.049, PMID 22537354.

25. Banerjee A, Lane DA, Torp-Pedersen C, Lip GYH. Net clinical benefit of new oral anticoagulants (dabigatran, rivaroxaban, apixaban) versus no treatment in a 'real world' atrial fibrillation population: a modelling analysis based on a nationwide cohort study. Thromb Haemost. 2012;107(3):584-9. doi: 10.1160/TH11-11-0784, PMID 22186961.

Article History: Submission Date : 02-10-2021; Revised Date : 29-10-2021; Acceptance Date : 13-11-2021.

Cite this article: Annie J, Doijad RC, James E. Prescription Pattern in the Management of Secondary Prevention of Stroke in Post Stroke Patients. Int. J. Pharm. Investigation. 2021;11(4):432-6. 